\title{
Uncommon Presentation of a Common Infection
}

\author{
Biswajit G Dastidar ${ }^{1}$, Shazia Gulshan ${ }^{2}$, Anindya Sarkar ${ }^{3}$, Arup K Halder ${ }^{4}$
}

\begin{abstract}
Introduction: Hemophagocytic lymphohistiocytosis $(\mathrm{HLH})$ is a clinical syndrome resulting from the immunological hyperactivation of macrophages. The condition can be triggered by an infection, malignancy, or autoimmune disease. Though the current literatures demonstrated a significant portion of sepsis patients are probably suffering from $\mathrm{HLH}$.

Case description: Here, we present a case who presented like features of gram-negative sepsis but prompt diagnosis of HLH and treatment saved the patient's life.

Conclusion: Hemophagocytic lymphohistiocytosis should be anticipated and recognized in fulminating sepsis-like conditions and promptly treated with steroids.

Keywords: Ferritin, Hemophagocytic lymphohistiocytosis, Salmonella, Sepsis.

The Journal of Medical Sciences (2020): 10.5005/jp-journals-10045-00159
\end{abstract}

\section{INTRODUCTION}

Hemophagocytic lymphohistiocytosis (HLH) is a clinical syndrome resulting from the immunological hyperactivation of macrophages. It causes the release of various proinflammatory cytokines like IL-1, IL-6, IL-8, and more. ${ }^{1,2}$ The histological feature of this condition is activated macrophages phagocyting other immune cells. The condition can be triggered by an infection, malignancy, or autoimmune disease. In 1984, Risdall reported this condition which often mimics sepsis. ${ }^{3}$ But for decades, and probably till today, this was believed to be extremely rare. Though the current literatures demonstrated a significant portion of sepsis patients are probably suffering from $\mathrm{HLH}^{4}{ }^{4,5}$ Here, we present a case who presented like features of gram-negative sepsis but prompt diagnosis of HLH and treatment saved the patient's life.

\section{Case Description}

This 27-year-old man was admitted with a history of fever and dry cough for 6 days along with diffuse pain abdomen and constipation. Initial evaluation revealed a right hypochondriac tenderness and dry coated tongue, the vital signs were normal except for the fever. He was COVID-19 negative. Initial investigations showed raised CRP, SGOT, SGPT, and the total leukocyte count was 5,000. After admission, the patient developed loose motion and IV saline was given. The initial working diagnosis was enteric fever and injection ceftriaxone was started. The next day an ultrasound abdomen was performed and revealed hepatosplenomegaly, gallbladder sludge, and distended bowel loops. But patient developed a very high spike of fever ( $104^{\circ}$ Fahrenheit) and tachycardia with diffuse pain in the abdomen; the procalcitonin level was much raised. So he was shifted to ICU after doing an abdominal CT scan, which revealed ileocecal thickening and multiple pericalyceal nodes. CRP, SGOT, and SGPT showed further rising trends but total leukocyte count decreased to 2,300. Severe sepsis was anticipated and antibiotics were switched to injection meropenem and teicoplanin instead of ceftriaxone. But patient continued to have extreme sinus tachycardia, tachypnea, restlessness, and confusion. The next day few reports showed rising CRP, SGOT, and SGPT with falling leukocyte count and platelets. Arterial blood gas showed
${ }^{1,3}$ Department of Medicine, Woodlands Hospital, Kolkata, West Bengal, India

${ }^{2}$ Department of Hematology, Woodlands Hospital, Kolkata, West Bengal, India

${ }^{4}$ Department of Pulmonology and Sleep, Institute of Sleep Sciences, Kolkata, West Bengal, India

Corresponding Author: Arup K Halder, Department of Pulmonology and Sleep, Institute of Sleep Sciences, Kolkata, West Bengal, India, e-mail: haldar.arup@yahoo.com

How to cite this article: Dastidar BG, Gulshan S, Sarkar A, et al. Uncommon Presentation of a Common Infection. J Med Sci 2020;6(4):68-69.

Source of support: Nil

Conflict of interest: None

respiratory alkalosis with preserved $\mathrm{PaO}_{2}: \mathrm{FiO}_{2}$. The lactate level was normal and urine culture was negative. Salmonella IgM was negative. Interestingly, LDH, ferritin $(32,095 \mathrm{ng} / \mathrm{mL})$, and triglyceride ( $662 \mathrm{mg} / \mathrm{dL}$ ) levels were way beyond normal. He had also features of rhabdomyolysis with elevated CPK $(18,856 \mathrm{IU} / \mathrm{L})$. The patient was reviewed by a hematologist and HLH was suspected.

The patient was given parenteral dexamethasone. By that night, the patient had lower temperature and tachycardia and for the first time since admission he felt well, and his mental function improved remarkably. The blood culture showed growth of Salmonella typhi sensitive to ceftriaxone and ofloxacin. So, meropenem and teicoplanin were stopped and shifted to sensitive antibiotics. C3 and C4 complement levels were raised and Epstein-Barr virus (EBV) serology was negative. The patient gradually became well with this regimen and blood parameters started improving. He was discharged 16 days after admission with tapering steroid doses. His final diagnosis was secondary HLH due to Salmonella typhi infection.

\section{Discussion}

Sepsis and HLH share many features like fever, cytopenias, hepatic involvement, disseminated intravascular coagulation 
Table 1: Investigations as per days

\begin{tabular}{llllllll}
\hline Day & D1 & D2 & D3 & D5 & D8 & D9 & D14 \\
\hline WBC & 5,000 & 2,300 & & 4,100 & & 6,300 \\
Platelet & & & 80,000 & 69,000 & & 90,000 & 325,000 \\
CRP & 79 & 280 & 312 & & & 51 & 1 \\
ALT & 100 & 160 & 168 & 195 & 196 & 201 & 187 \\
AST & 144 & 644 & 926 & 1,321 & 1,101 & 721 & 64 \\
Ferritin & & & 32,095 & 37,301 & & 4,712 & 1,133 \\
CPK & & & & 18,856 & & & 129 \\
\hline
\end{tabular}

(DIC). ${ }^{6}$ But like HLH which is often triggered by viral infections, sepsis is caused by bacterial or fungal infections. The most common infectious trigger of HLH is-EBV, cytomegalovirus (CMV), parvovirus, herpes simplex virus, varicella-zoster virus, measles virus, human herpesvirus $6, \mathrm{H} 1 \mathrm{~N} 1, \mathrm{HIV}$, SARS-CoV2. ${ }^{7}$ Occasionally bacterial infections like Brucella, gram-negative bacteria like Salmonella or Mycobacterium tuberculosis or parasites like leishmaniasis, malaria or fungus may also give rise to $\mathrm{HLH}^{7}$ The following modified criteria are sufficient to diagnose $\mathrm{HLH}$ : three of four clinical findings (fever, splenomegaly, cytopenias, hepatitis) plus abnormality of one of four immune markers (hemophagocytosis, increased ferritin, hypofibrinogenemia, absent or very decreased NK cell function). ${ }^{8}$ Unfortunately, there are no laboratory tests to distinguish between sepsis and $\mathrm{HLH}$, but extremely high ferritin and elevated LDH are more common in $\mathrm{HLH}^{9}{ }^{9}$ Bone marrow examination to demonstrate hemophagocytosis in HLH is not often feasible in acutely ill patients nor is required to diagnose HLH. ${ }^{10}$ Treatment of underlying condition and prompt treatment with steroid are life-saving in this condition.

Salmonella as a cause of HLH is not common, but some case reports are there in the medical literature. ${ }^{11}$ The uniqueness of the current case lies here; where the patient presented as a case of fulminating gram-negative sepsis, but not responding to antibiotics alone. A timely administration of steroids, after quick anticipation of $\mathrm{HLH}$, helped to save a precious life. ${ }^{12}$ Another important aspect was the presence of rhabdomyolysis in Salmonella is also very rare which was observed in the present case. This was not a part of HLH criteria and was thought to be a separate complication of invasive Salmonella infection.

\section{Conclusion}

Hemophagocytic lymphohistiocytosis can closely mimic sepsis and is not uncommon. This disease should be anticipated and recognized in fulminating sepsis-like conditions and promptly treated with steroids (Table 1).

\section{REFERENCES}

1. Janka GE, Lehmberg K. Hemophagocytic syndromes--an update. Blood Rev 2014;28(4):135-142. DOI: 10.1016/j.blre.2014.03.002.

2. Park HS, Kim DY, Lee JH, et al. Clinical features of adult patients with secondary hemophagocytic lymphohistiocy tosis from causes other than lymphoma: an analysis of treatment outcome and prognostic factors. Ann Hematol 2012;91(6):897-904. DOI: 10.1007/s00277-0111380-3.

3. Rosado FGN, Kim AS. Hemophagocytic lymphohistiocytosis: an update on diagnosis and pathogenesis. Am J Clin Pathol 2013;139(6):713-727. DOI: 10.1309/AJCP4ZDKJ4ICOUAT.

4. Lachmann G, Spies C, Schenk T, et al. Hemophagocytic lymphohistiocytosis: potentially underdiagnosed in intensive care units. Shock 2018;50(2):149-155. DOI: 10.1097/SHK.0000000000001048.

5. Kumar V, Sharma B, Nigam AS. Case series of hemophagocytic lymphohistiocytosis from a tertiary care centre: an underdiagnosed entity. Turk Patoloji Derg 2019;35(3):207-212. DOI: 10.5146/ tjpath.2018.01456.

6. Machowicz R, Janka G, Wiktor-Jedrzejczak W. Similar but not the same: differential diagnosis of HLH and sepsis. Crit Rev Oncol Hematol 2017;114:1-12. DOI: 10.1016/j.critrevonc.2017.03.023.

7. George MR. Hemophagocytic lymphohistiocytosis: review of etiologies and management. J Blood Med 2014;5:69-86. DOI: 10.2147/ JBM.S46255.

8. Jordan MB, Allen CE, Greenberg J, et al. Challenges in the diagnosis of hemophagocytic lymphohistiocytosis: recommendations from the North American consortium for histiocytosis (NACHO). Pediatr Blood Cancer 2019;66(11):e27929. DOI: 10.1002/pbc.27929.

9. Allen CE, Yu X, Kozinetz CA, et al. Highly elevated ferritin levels and the diagnosis of hemophagocytic lymphohistiocytosis. Pediatr Blood Cancer 2008;50(6):1227-1235. DOI: 10.1002/pbc.21423.

10. Ho C, Yao X, Tian L, et al. Marrow assessment for hemophagocytic lymphohistiocytosis demonstrates poor correlation with disease probability. Am J Clin Pathol 2014;141(1):62-71. DOI: 10.1309/ AJCPMD5TJEFOOVBW.

11. Singh ZN, Rakheja D, Yadav TP, et al. Infection-associated haemophagocytosis: the tropical spectrum. Clin Lab Haematol 2005;27(5):312-315. DOI: 10.1111/j.1365-2257.2005.00717.x.

12. Non LR, Patel R, Esmaeeli A, et al. Typhoid fever complicated by hemophagocytic lymphohistiocytosis and rhabdomyolysis. Am J Trop Med Hyg 2015;93(5):1068-1069. DOI: 10.4269/ajtmh.15-0385. 